\title{
Efficient Inference in Large Conditional Random Fields
}

\author{
Trevor Cohn ${ }^{\star}$
}

School of Informatics, University of Edinburgh, EH8 9LE, United Kingdom

\begin{abstract}
Conditional Random Fields (CRFs) are widely known to scale poorly, particularly for tasks with large numbers of states or with richly connected graphical structures. This is a consequence of inference having a time complexity which is at best quadratic in the number of states. This paper describes a novel parameterisation of the CRF which ties the majority of clique potentials, while allowing individual potentials for a subset of the labellings. This has two beneficial effects: the parameter space of the model (and thus the propensity to over-fit) is reduced, and the time complexity of training and decoding becomes sub-quadratic. On a standard natural language task, we reduce CRF training time four-fold, with no loss in accuracy. We also show how inference can be performed efficiently in richly connected graphs, in which current methods are intractable.
\end{abstract}

\section{Introduction}

Conditional random fields (CRFs) [1] are probabilistic models for labelling structured data. CRFs are undirected graphical models which define a conditional distribution over labellings given an observation. They allow the use of arbitrary, overlapping and nonindependent features, avoiding strong independence assumptions over the observation which are typically required by generative models. CRFs have proven very successful in natural language processing [1/2]3].

However, CRFs have typically only been applied to relatively small tasks - those with small label sets, few training instances and using a simple chain. This is because inference in a linear chain CRF has a time complexity quadratic in the number of labels. The complexity is even greater for graphs with larger cliques. This serves to limit the model's scalability to large tasks and prevents the use of richly connected graphs.

This paper describes a novel mechanism that can reduce the complexity of inference. We constrain the labellings considered in each feature function, such that the functions can detect only a relatively small set of labellings. The remaining labellings can be detected only en masse, with the features unable to discriminate between each labelling. As such, the clique potentials share the same tied structure, containing many identical values. The sum-product and max-product algorithms can exploit the tied potentials for a significant reduction in runtime, resulting in faster training and decoding.

In many language tasks there are few truly useful labellings; these can be easily enumerated. Accordingly, tying the potentials of all remaining labellings should not noticeably reduce the modelling ability of the CRF, and may make better use of sparse data. We show how this technique can be used to reduce the training and decoding times for part-of-speech tagging, while achieving state-of-the-art results. We also demonstrate that richly connected graphs can be used tractably to perform semantic role labelling.

\footnotetext{
* Previously from the Department of CSSE, University of Melbourne, VIC 3010, Australia.
} 


\section{Conditional Random Fields}

CRFs are undirected graphical models which define the conditional probability of an assignment of output labels (states) given an input observation [1]. The joint probability density function of the labelling, $\mathbf{s}$, given the input observation, $\mathbf{o}$, is given by:

$$
p_{\Lambda}(\mathbf{s} \mid \mathbf{o})=\frac{1}{Z(\mathbf{o})} \prod_{c \in \mathcal{C}} \psi_{c}\left(\mathbf{s}_{c}, \mathbf{o}\right)=\frac{1}{Z(\mathbf{o})} \exp \sum_{c \in \mathcal{C}} \sum_{k} \lambda_{k} f_{k}\left(c, \mathbf{s}_{c}, \mathbf{o}\right)
$$

where $\mathcal{C}$ is the set of cliques and $\psi_{c}$ are the potential functions, which map the clique labelling and observation into a positive scalar. $Z(\mathbf{o})$ is the partition function which ensures that $p$ is correctly normalised. We use the maximum entropy principle to define the potentials, $\psi_{c}\left(\mathbf{s}_{c}, \mathbf{o}\right)=\exp \sum_{k} \lambda_{k} f_{k}\left(c, \mathbf{s}_{c}, \mathbf{o}\right)$, where $\lambda_{k}$ are the parameters of the model, and the functions $f_{k}$ are feature functions. The feature functions are usually binary valued, and combine an observational test with a labelling test. For example, a typical feature for POS tagging might detect a word suffix of "ing" coupled with a VBG label. The features typically combine observation tests with label unigrams or bigrams.

Training typically involves finding the parameters which maximise the log-likelihood of an i.i.d. fully observed training set, $\left(\mathbf{o}^{(i)}, \mathbf{s}^{(i)}\right)_{i=1}^{N}$, consisting of (observation, labelling) pairs. This is subject to a prior over the parameter values, $p(\Lambda)$, yielding an objective of the form:

$$
\mathcal{O}=\sum_{i} \log p_{\Lambda}\left(\mathbf{s}^{(i)} \mid \mathbf{o}^{(i)}\right)+\log p(\Lambda) .
$$

The parameters which maximise $\mathcal{O}$ cannot be found analytically; instead we need to employ iterative methods. Gradient descent methods have proven the most efficient [4|2]; these require repeated evaluation of objective (2) and its derivatives with respect to each parameter $\lambda_{k}$. The partition function and the derivatives prove costly to calculate requiring the marginal distributions over each clique. These marginals can be computed using sum-product belief propagation (BP) when the graph is acyclic, or loopy $\mathrm{BP}$, an iterative approximation method [5], otherwise. This has a time complexity of $O\left(E S^{C}\right)$ where $E$ are the number of edges in the graph, $S$ is the number of labels and $C$ is the size of the maximal clique.

Decoding uses max-product belief propagation [5] to solve $\mathbf{s}^{*}=\arg \max _{\mathbf{s}} p(\mathbf{s} \mid \mathbf{o})$. As with the sum-product algorithm, the time complexity is $O\left(E S^{C}\right)$ : at least quadratic in the label set.

\section{Tied Potentials}

We propose the use of tied potentials to simplify both the sum- and max- product algorithms, and thus training and decoding. Firstly we make the standard assumption that all the features have the form $f_{k}\left(c, \mathbf{s}_{c}, \mathbf{o}\right)=g_{k}(c, \mathbf{o}) h_{k}\left(\mathbf{s}_{c}\right)$, where $g$ detects features of the observation and $h$ detects features of the labelling. We then partition the full labelling space into a number of disjoint sets, $S^{K}=\bigcup_{i=1}^{N} \mathcal{S}_{i}$, where $S$ is the labels set and $K$ is the factor size. We constrain the functions $h_{k}$ to detect only whether the labelling is in 
one of these sets. I.e. the $h_{k}$ functions are limited to testing set membership rather than testing for exact label configurations. These sets are chosen a priori, and typically conflate sets of label configurations which are better modelled together than individually. We assume the last of these sets $\mathcal{S}_{N}$ is the largest, which call the remaining labellings. We call the labellings in the other sets the selected labellings.

These constrained features lead to the potentials with the form:

$$
\psi_{f}\left(\mathbf{s}_{f}, \mathbf{o}\right)=\sum_{i} \omega_{i} \llbracket \mathbf{s}_{f} \in \mathcal{S}_{i} \rrbracket \quad \text { where } \omega_{i}=\exp \sum_{k \in \mathcal{K}_{i}} \lambda_{k} g_{k}(f, \mathbf{o})
$$

where $\llbracket \cdot \rrbracket$ is one if the test succeeds and zero otherwise and $\mathcal{K}_{i}$ indexes the features which detect a labelling in $\mathcal{S}_{i}$.

\subsection{Sum-Product}

Sum-product belief propagation (BP) can exploit the tied potential structure in (3). We describe its application to BP in factor graphs [5], a convenient representation when there are cliques over more than three nodes. BP requires messages to be sent over each edge in the factor graph in both directions. The marginal distributions are calculated from these messages. The messages sent from nodes to factors remain unchanged, while the messages from factors to nodes are updated to reflect the tied potentials:

$$
m_{f \rightarrow n_{i}}\left(s_{i}\right)=\sum_{\mathbf{s}_{f}^{\prime}: s_{i}^{\prime}=s_{i}} \omega_{N} M\left(\mathbf{s}_{f}^{\prime}\right)+\sum_{j=1}^{N-1} \sum_{\mathbf{s}_{f}^{\prime} \in \mathcal{S}_{j}: s_{i}^{\prime}=s_{i}}\left(\omega_{j}-\omega_{N}\right) M\left(\mathbf{s}_{f}^{\prime}\right)
$$

where $M\left(\mathbf{s}_{f}^{\prime}\right)=\prod_{n_{j} \in \mathcal{N}(f) \backslash n_{i}} m_{n_{j} \rightarrow f}\left(s_{j}^{\prime}\right)$ is the product of incoming messages. The above formulation introduces the default (tied) potential, $\omega_{N}$, for every possible label configuration, which is offset by the potential difference, $\omega_{j}-\omega_{N}$, for every selected configuration. If we assume that messages are normalised to sum to one, we can further simplify the first term in (4) to $\omega_{N}$. Here the summation over the full space of labellings is avoided; it is instead replaced by sums over only the selected labellings. This results in a time complexity for sum-product inference of $O(E T)$ where $T$ are the number of selected labellings, regardless of the factor size 1 The cost of loopy BP cannot be bounded in general; in this case the complexity applies to a single round. The factor marginals can be calculated from the messages using the same trick from (4) to exploit the tied potential values. This allows the efficient calculation of the normalisation constant for each marginal distribution without explicitly enumerating every labelling.

\subsection{Max-Product}

Max-product belief propagation is used for decoding. The most expensive message is that going from factor to node:

$$
m_{f \rightarrow n_{i}}\left(s_{i}\right)=\max _{j=1 \ldots N} \omega_{j} \max _{\mathbf{s}_{f}^{\prime} \in \mathcal{S}_{j}: s_{i}^{\prime}=s_{i}} M\left(\mathbf{s}_{f}^{\prime}\right)
$$

\footnotetext{
${ }^{1}$ Larger factors will require more computation, as the number of messages in the product $M\left(\mathbf{s}_{f}\right)$ will increase. However, this effect is linear in the factor size, not exponential.
} 
In order to calculate this maximum, we use a dynamic program over the set of selected labellings. We construct a trie over the selected labellings, as illustrated in Fig. 1 This trie shows three selected sets of labellings and therefore four distinct potentials one for each selected set and one for the remaining set. The paths leading to remaining labellings are omitted from the tree, shown by dotted edges. By traversing this tree, we can maximise over both the selected labellings and the remaining labelling.

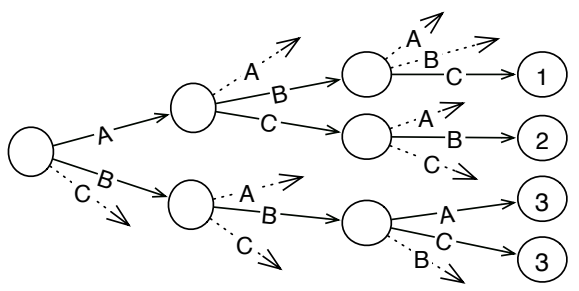

Fig. 1. An example trie with four selected labellings. The leaves are annotated with their potential index, and the dotted lines denote omitted paths leading to remaining labellings.

Maximisation uses a preorder traversal, recording the score for the current partial path. Once we reach a leaf, we have found a selected labelling; its score is the product of its potential $\omega_{i}$ and the leaf score. At each stage in our traversal we may traverse off the trie by following a dotted link, after which no path can yield a selected labelling. Therefore we can maximise the score for subsequent states without constraints. This maximum is simply $\omega_{N}$ if we ensure that messages are normalised such that the maximum message value is 1 .

This approach reduces the search space down to the number of nodes in the tree (with an additional fringe). This can be further reduced by traversing the tree in a bestfirst manner, using $A^{*}$ search to guide us towards branches of the tree in which we expect high scores. As a simple heuristic we use the product of the node score and the maximum potential value, which often takes a very small number of steps. This does not result in a reduction in the complexity bound, but in practice it considerably reduces the run-time.

\section{Experiments}

Two experiments were conducted on tasks which test the scalability of the CRF. The first task is POS tagging, which has a large label set and many training instances. In this task, standard CRF training using a linear chain is only just possible with modern hardware, and we show how this can be made considerably faster. The second task is semantic role labelling (SRL), in which we use richly connected cyclic graphs with many large factors. For such graphs, standard loopy belief propagation is impossible due to the large factor size. We show that feature tying can be used to make training and decoding possible for such graphs. 


\subsection{Part-of-Speech Tagging}

The first experiment entailed tagging words with part-of-speech labels, which was modelled with a chain CRF. We used the Penn Treebank III [6], training on sections 2-21, using section 24 for development and section 23 for testing. There are 45,110 training sentences, a total of 1,023,863 tokens and 45 labels. The timing experiments used only the first 1,000 training sentences, while the performance experiments used the full set.

The observation features included word identity, prefixes and suffixes, whether the word contains a number, uppercase letter or a hyphen, nearby words at relative positions $-2,-1,+1$ and +2 , and the word shape [7]. The word identity and default (always true) observation feature were conjoined with label pairs over pair-wise cliques, while all observation features were conjoined with single node clique labels to form the feature set. Only features seen at least once in training were included.

Timing. Three sets of selected pair-wise labellings were derived from the reduced training set: those transitions occurring at least 50 times, 10 times or once. Each selected transition labelling was modelled with a separate potential, while the remaining transitions were tied. Note that the tied transition features were used alongside standard single node features; the unigram features allow the model to disambiguate between the various tied bigrams. Table 1 a shows the results when the models were trained and used for decoding both with the tied-potential optimisations (labelled 'tied') and without ('dense').

The training times for the tied optimised models are considerably lower than the dense model, despite taking more iterations to converge. The tied decoding times increase with the number of selected labellings, and are mostly competitive with the dense decoding, although the cost of constructing and traversing the trie eliminates speed gains when there are many selected labellings. The accuracies of the models trained with each transition threshold were almost identical: clearly no modelling power was sacrificed by excluding rare transitions. Moreover, using ML training, the thresholded transitions (at 10) yielded significantly better accuracy than the complete model (using the McNemar matched-pairs test at $0.1 \%[8]$ ), indicating that the tying of features is smoothing the model.

Table 1. Part-of-speech tagging timing and performance results

(a) Timings using training subset

\begin{tabular}{|r|ccc|}
\hline Threshold & 50 & 10 & 1 \\
\#-selected & 109 & 302 & 770 \\
\hline Train - tied & $1635 \mathrm{~s}$ & $2084 \mathrm{~s}$ & $3664 \mathrm{~s}$ \\
Train - dense & $5144 \mathrm{~s}$ & $4326 \mathrm{~s}$ & $4222 \mathrm{~s}$ \\
Decode - tied & $21 \mathrm{~s}$ & $33 \mathrm{~s}$ & $51 \mathrm{~s}$ \\
Decode - dense & $36 \mathrm{~s}$ & $26 \mathrm{~s}$ & $24 \mathrm{~s}$ \\
MLE accuracy & $91.93 \%$ & $92.47 \%$ & $91.99 \%$ \\
MAP accuracy & $93.09 \%$ & $93.31 \%$ & $93.33 \%$ \\
\hline
\end{tabular}

(b) Accuracy using full training set

\begin{tabular}{|r|ccc|}
\hline Threshold & 1000 & 500 & 100 \\
\#-selected & 102 & 227 & 507 \\
\hline Accuracy dev. & $96.86 \%$ & $96.91 \%$ & $96.91 \%$ \\
Accuracy test & $97.13 \%$ & $97.22 \%$ & $97.24 \%$ \\
Iteration time & $700 \mathrm{~s}$ & $1160 \mathrm{~s}$ & $2072 \mathrm{~s}$ \\
\hline
\end{tabular}


Performance. The tied models were then applied to the full training set, using thresholds of 1000, 500 and 100. Lowering this threshold considerably increased the number of selected labellings and thus the run-time requirements for no tangible gain; therefore we stopped at 100 . We could not train a CRF using the standard (dense) training on the full task for comparison, due to its high runtime requirements. The performance of these models on the development and test sets are shown in Table $1 \mathrm{~b}$ A Gaussian prior was used with a zero mean and unit variance. Once again, we see that the performance is quite similar across all thresholds (the 500 and 100 results are insignificantly different), showing that a simple frequency based selection policy is adequate. As expected, the per-iteration cost of training increases with the number of selected labellings. Our best accuracy equals that of [9], to our knowledge the best result to date, although we used a different training and test split. Unlike [9], our model achieved this result without using features over tag 3-grams and 4-grams.

\subsection{Semantic Role Labelling}

The second experiment demonstrates how tied-potentials can be used in order to allow efficient inference on graphs with very large factors. Semantic role labelling (SRL; [10]) is the task of identifying which groups of words act as arguments to a given verbal predicate, and what role they fill - i.e. agent, patient, etc. We adopt the CoNLL task specification [11], where a predicted syntactic parse tree is provided.

We treat the task as one of labelling each word with a role label which indicates that the word is part of a constituent with this role. The syntax tree specifies the set of syntactic constituents, and each is used as a factor, connected to the node for all words in its yield. Figure 2 shows an example sentence and some induced syntactic factors. The features for a factor were constrained to select only homogeneous labellings over its nodes. Therefore only $|S|$ labellings $(|S|$ is the number of labels) are detected instead of an exponential number in $|S|$. These features can bias the model towards choosing uniform argument labels for all nodes in an argument constituent, while still allowing overlapping constituents to compete for dominance over the labelling. In addition, we introduced adjacency factors linking adjacent constituents (e.g., linking 'I' and 'the' to join $\mathrm{NP}_{1}$ and $\mathrm{NP}_{2}$ ), representing a first order Markov assumption between adjacent argument candidates.
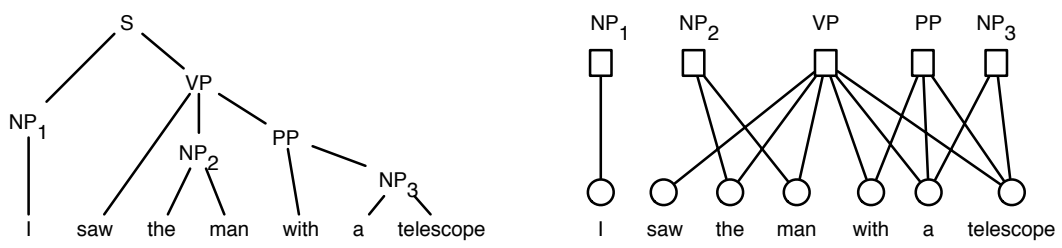

Fig. 2. Example SRL factor graph, showing the syntactic parse and an induced factor graph

The observation features were taken from [12], which included syntactic paths, head words, syntactic category, etc. We also used simple pruning [13] to remove irrelevant 
nodes from the parse tree before creating the factor graph. For further simplification, the factor graph was shrunk by merging adjacent nodes which were both members of the same set of syntactic factors, forcing them to be labelled together. Despite these simplifications, most graphs were cyclic and had large factors.

This model was trained on the proposition-bank corpus [10], using the CoNLL 2005 shared task dataset [11]. We considered only the core argument labels (A0 - A5), which roughly map to the roles of agent, patient, theme, etc. Sections 2-21 were used for training, and section 24 for development. The $\mathrm{F}_{1}$ score on the development set was $76.56 \%$. These results can be roughly compared to [14], who applied (constrained) CRFs to the SRL argument labelling task. They report an $F_{1}$ score of $74.49 \%$, although on a different version of the data set.

\section{Related Work}

Siddiqi and Moore present a similar approach for fast inference in hidden Markov models [15]. They encode the state transition matrix by preserving the top $K$ transitions from a given state, while sharing the remaining probability mass evenly between all other transitions from that state. They present optimised versions of the forwardbackward algorithm, Viterbi and EM training, all which have considerably reduced complexity. Our work applies a similar method to log-linear undirected graphical models, showing how feature constraints can produce a similar 'mostly-constant' transition matrix (the tied potentials). While Siddiqi and Moore's model requires the specification of the number of dense outgoing transitions, $K$, our model instead requires explicit enumeration of these configurations. We show how this transition matrix structure can readily be exploited in general (possibly exact) belief propagation for factor graphs, as opposed to just forward-backward and Viterbi on chains.

Other approximate inference techniques have been used to reduce CRF training time, such as beam-search during sum-product or max-product inference [16], or using the voted perceptron algorithm [17]. These approximations are orthogonal to our approach, and could feasibly be used together for further gains. Our approach allows CRF inference in graphs with very large factors. For these graphs, standard training - and approximate training - is intractable.

Similar feature based optimisation has been used for training of log-linear language models [18]. For this task, the label space is extremely large (the vocabulary of words), and the optimisations presented allow efficient grouping of labels with similar feature sets. Our method also groups features, but in the context of belief propagation for globally normalised log-linear models.

\section{Conclusion}

We have shown how the use of tied potentials can reduce the time complexity of inference for a conditional random field. In a chain, this reduces the complexity to be sub-quadratic in the number of states, making training and decoding faster for many currently difficult tasks. Even for graphs with considerably larger cliques, inference remains tractable, allowing CRFs to be applied to previously impossible tasks. We have 
shown in our experiments how a small set of selected labellings can be used to reduce training time without sacrificing performance, equalling the state-of-the-art accuracy for POS tagging. We also showed how this technique allows CRFs to be used with richer more densely connected graphs for semantic role labelling.

Acknowledgements. Special thanks to Miles Osborne, Steven Bird, Phil Blunsom and Andrew Smith for their insightful feedback.

\section{References}

1. Lafferty, J., McCallum, A., Pereira, F.: Conditional random fields: Probabilistic models for segmenting and labelling sequence data. In: Proceedings of ICML. (2001) 282-289

2. Sha, F., Pereira, F.: Shallow parsing with conditional random fields. In: Proceedings of HLT-NAACL. (2003) 213-220

3. Cohn, T., Smith, A., Osborne, M.: Scaling conditional random fields using error-correcting codes. In: Proceedings of ACL. (2005)

4. Malouf, R.: A comparison of algorithms for maximum entropy parameter estimation. In: Proceedings of CoNLL. (2002) 49-55

5. Kschischang, F., Frey, B., Loeliger, H.A.: Factor graphs and the sum-product algorithm. IEEE Trans. Inform. Theory 47 (2001) 498-519

6. Marcus, M., Kim, G., Marcinkiewicz, M.A., MacIntyre, R., Bies, A., Ferguson, M., Katz, K., Schasberger, B.: The Penn Treebank: Annotating predicate argument structure. In: Proceedings of ARPA Human Language Technology Workshop. (1994)

7. Collins, M.: Ranking algorithms for named entity extraction: Boosting and the voted perceptron. In: Proceedings of ACL. (2002) 489-496

8. Gillick, L., Cox, S.: Some statistical issues in the comparison of speech recognition algorithms. In: Proceedings of ICASSP. (1989) 532-535

9. Toutanova, K., Klein, D., Manning, C., Singer, Y.: Feature rich part-of-speech tagging with a cyclic dependency network. In: Proceedings of HLT-NAACL. (2003) 252-259

10. Palmer, M., Gildea, D., Kingsbury, P.: The proposition bank: An annotated corpus of semantic roles. Computational Linguistics 31(1) (2005) 71-105

11. Carreras, X., Màrquez, L.: Introduction to the CoNLL-2005 Shared Task: Semantic Role Labeling. In: Proceedings of CoNLL. (2005) 152-164

12. Pradhan, S., Hacioglu, K., Krugler, V., Ward, W., Martin, J., Jurafsky, D.: Support vector learning for semantic argument classification. Machine Learning journal, Special issue on Speech and Natural Language Processing 60(1) (2005) 11-39

13. Xue, N., Palmer, M.: Calibrating features for semantic role labeling. In: Proceedings of EMNLP. (2004) 88-94

14. Roth, D., Yih, W.: Integer linear programming inference for conditional random fields. In: Proceedings of ICML. (2005) 737-744

15. Siddiqi, S., Moore, A.: Fast inference and learning in large-state-space HMMs. In: Proceedings of ICML. (2005) 800-807

16. Pal, C., Sutton, C., McCallum, A.: Sparse forward-backward using minimum divergence beams for fast training of conditional random fields. In: Proceedings of ICASSP. (2006)

17. Roark, B., Saraclar, M., Collins, M., Johnson, M.: Discriminative language modeling with conditional random fields and the perceptron algorithm. In: Proceedings of ACL. (2004) $48-55$

18. Wu, J., Khudanpur, S.: Efficient training methods for maximum entropy language modelling. In: Proceedings of the ICSLP. (2000) 114-117 